\title{
Risk Factors for Middle East Respiratory Syndrome Coronavirus Infection among Healthcare Personnel
}

\author{
Basem M. Alraddadi, Hanadi S. Al-Salmi, Kara Jacobs-Slifka, Rachel B. Slayton, \\ Concepcion F. Estivariz, Andrew I. Geller, Hanan H. Al-Turkistani, Sanaa S. Al-Rehily, \\ Haleema A. Alserehi, Ghassan Y. Wali, Abeer N. Alshukairi, Esam I. Azhar, Lia Haynes, \\ David L. Swerdlow, John A. Jernigan, ${ }^{1}$ Tariq A. Madani ${ }^{1}$
}

\begin{abstract}
Healthcare settings can amplify transmission of Middle East respiratory syndrome coronavirus (MERS-CoV), but knowledge gaps about the epidemiology of transmission remain. We conducted a retrospective cohort study among healthcare personnel in hospital units that treated MERS-CoV patients. Participants were interviewed about exposures to MERS-CoV patients, use of personal protective equipment, and signs and symptoms of illness after exposure. Infection status was determined by the presence of antibodies against MERS-CoV. To assess risk factors, we compared infected and uninfected participants. Healthcare personnel caring for MERS-CoV patients were at high risk for infection, but infection most often resulted in a relatively mild illness that might be unrecognized. In the healthcare personnel cohort reported here, infections occurred exclusively among those who had close contact with MERS-CoV patients.
\end{abstract}

M iddle East respiratory syndrome coronavirus (MERS$\mathrm{CoV}$ ), first identified in 2012, has emerged as a cause of severe acute respiratory illness in humans. As of May 1, 2016, a total of 1,728 laboratory-confirmed cases, including 624 deaths, have been reported globally (1). All reported cases have been directly or indirectly linked to countries in or near the Arabian Peninsula, including a recent outbreak in South Korea resulting from a single imported case in a person with history of travel to the Middle East $(2,3)$. Increasing evidence suggests that dromedary camels

Author affiliations: King Faisal Specialist Hospital and Research Center, Jeddah, Saudi Arabia (B.M. Alraddadi, H.S. Al-Salmi,

H.H. Al-Turkistani, S.S. Al-Rehily, H.A. Alserehi, G.Y. Wali, A.N. Alshukairi); Ministry of Health, Jeddah (B.M. Alraddadi, E.I. Azhar, T.A. Madani); Centers for Disease Control and Prevention, Atlanta, Georgia, USA (K. Jacobs-Slifka, R.B. Slayton, C.F. Estivariz, A.I. Geller, L. Haynes, D.L. Swerdlow,

J.A. Jernigan); King Abdulaziz University, Jeddah (E.I. Azhar, T.A. Madani)

DOI: http://dx.doi.org/10.3201/eid2211.160920 are a natural host for MERS-CoV and that camel-to-human transmission can occur, initiating short chains of human-tohuman transmission (4-7). Numerous questions about the epidemiology of MERS-CoV remain unanswered.

Healthcare settings are important amplifiers of transmission $(6,8,9)$. A 2014 case series of 255 MERS-CoV infections in Saudi Arabia found that 31\% of cases occurred among healthcare personnel (HCP), and among casepatients who were not HCP, $87.5 \%$ had recent healthcare exposure (9). Current MERS-CoV infection control recommendations are based on experience with other viruses rather than on a complete understanding of the epidemiology of MERS-CoV transmission $(10,11)$.

The World Health Organization recently issued an urgent call for studies to better understand risk factors for infection and transmission (12). Published case series of healthcare-associated MERS-CoV infections have major limitations, including lack of control groups and lack of serologic confirmation of infection status, leaving wide knowledge gaps, such as mode of and risk factors for transmission in healthcare settings, attack rate among HCP, and spectrum of illness for MERS-CoV infection (13). To address these gaps, we retrospectively studied MERS-CoV infection among a cohort of HCP in a hospital in Saudi Arabia.

\section{Methods}

The study was conducted at King Faisal Specialist Hospital and Research Center (Jeddah, Saudi Arabia) during May-June 2014. This multispecialty hospital has 360 beds, including an 18-bed medical intensive care unit (MICU) and a 38-bed emergency department (ED). Seventeen patients with confirmed MERS-CoV infection were in the hospital during March 24-May 3, 2014. The hospital had no cases of MERS-CoV before March 24, 2014. All patients with suspected or confirmed MERS$\mathrm{CoV}$ infection were placed in private rooms equipped

${ }^{1}$ These authors contributed equally to this article. 
with negative pressure ventilation. Patients in whom MERS-CoV infection was not suspected initially were transferred to negative-pressure rooms as soon as the diagnosis was suspected or confirmed. During the outbreak, all HCP who had contact with MERS-CoV cases were screened for symptoms and underwent testing for MERSCoV RNA by real-time reverse transcription PCR (rRTPCR) of nasopharyngeal swab specimens.

We assessed risk factors for a case, defined as a MERSCoV antibody-positive serum sample from an HCP, among 3 cohorts of HCP. Two cohorts, 1 each from the ED and MICU, comprised all HCP who worked in those hospital units during March 24-May 14, 2014, the period during which those units treated patients known to have MERS$\mathrm{CoV}$ infection. In addition, we included a cohort of all HCP who worked in a unit (neurology) that was not known to house any MERS-CoV patients during the study period.

Every healthcare worker in each cohort was recruited to enroll. Participants provided a serum sample and were interviewed by trained study personnel using a standardized questionnaire. Although HCP were from different cultural, language, and educational backgrounds, all spoke English fluently. All questionnaires were conducted in English. Interviews were conducted during May 28-July 10, 2014. In addition to age, sex, occupation, and co-morbidities, we collected information on signs, symptoms, and treatment from March 31, 2014, through the day of interview. Contacts with MERS-CoV patients were described, including patient care activities, duration of contact, and exposure to body fluids. Information about infection control training and use of personal protective equipment (PPE) during encounters with MERS-CoV patients was collected. We assessed exposures outside the hospital, including household exposures to persons with MERS-CoV, contact with animals, and travel.

Serum samples were screened for antibodies against MERS-CoV (Hu/Jordan-N3/2012) nucleocapsid (N) protein by ELISA. The recombinant MERS-CoV N indirect ELISA was developed by using a modified version of the HKU5.2 N ELISA previously described (13) Serum was considered positive when the optical density values were $\geq 0.36$ (mean absorbance $405 \mathrm{~nm}$ of serum from US blood donors $+3 \mathrm{SD}$ ) with an assay specificity of $98.1 \%$ $(544 / 555)$. Samples that were positive by ELISA were confirmed by immunofluorescence assay, microneutralization assay, or both (14). A positive serologic test result required confirmation by immunofluorescence assay or microneutralization assay. HCP whose serum sample tested positive for MERS-CoV antibodies were considered to have evidence of MERS-CoV infection (case-HCP); seronegative persons were considered uninfected.

We analyzed data using SAS version 9.3 (SAS Institute, Cary, NC, USA). As appropriate, we compared dichotomous variables using $\chi^{2}$ and Fisher exact tests. Cochran-Armitage tests for trend were used for ordinal variables. We performed multivariate logistic regression with backward stepwise elimination for exposures with univariate $p \leq 0.2$. Variables with $p \leq 0.1$ were retained in the final generalized linear model using a logit link to estimate risk.

We obtained written informed consent from all participants. The Institutional Review Board of the King Faisal Specialist Hospital and Research Centre approved the study.

\section{Results}

Of $363 \mathrm{HCP}$ eligible for the MICU (178 HCP), ED (137 HCP), and neurology unit (48 HCP) cohorts, 292 (80.4\%) HCP were enrolled: $131(73.5 \%)$ from the MICU, 127 (92.7\%) from the ED, and $34(70.8 \%)$ from the neurology unit. Of the 292 enrolled persons, 9 were excluded because serum specimens were unavailable.

For study participants who worked in units that treated MERS-CoV patients, the attack rate was $8.0 \%(20 / 250)$ and varied by hospital unit: MICU, $11.7 \%$ (15/128); ED, $4.1 \%(5 / 122)$. (The attack rate in the neurology unit, where no known MERS-CoV patients were treated, was $0 \%$ [0/33].) Attack rates in the MICU and ED also varied by occupation; radiology technicians had the highest attack rate $(29.4 \%$ [5/17]), followed by nurses $(9.4 \%$ [13/138]), respiratory therapists $(3.2 \%[1 / 31])$, and physicians $(2.4 \%$ [1/41]). No clerical staff (7 participants) or patient transporters (14 participants) were seropositive. Most participants $(64.4 \%$ [161/250]) were female; attack rate did not differ by sex (male $7.9 \%$, female $8.1 \%$; $p=0.95$ ). The mean age of seropositive HCP was 40 years (range 29-59 years) and of seronegative HCP 37 years (range 18-66 years).

The most common manifestations of illness among case-HCP were muscle pain, fever, headache, and dry cough (Table 1). These signs and symptoms, along with shortness of breath, occurred significantly more often among seropositive than among seronegative HCP. Seropositive HCP were also more likely to report gastrointestinal symptoms $(\mathrm{p}<0.001)$. Of the 20 case-HCP, $3(15 \%)$ were asymptomatic, $12(60 \%)$ had mild illness (symptomatic illness not requiring hospital admission), 2 (10\%) had moderate illness (required hospital admission but not mechanical ventilation), and 3 (15\%) had severe illness (required mechanical ventilation). All case-HCP survived, and all had been previously tested for MERS-CoV by rRTPCR of nasopharyngeal swab specimens, but only $5(25 \%)$ rRT-PCRs were positive.

Nineteen $(95 \%)$ of 20 case-HCP reported having been in the same room as or within 2 meters of a patient known to be infected with MERS-CoV. The 1 seropositive HCP who had no MERS-CoV patient contact reported being in an automobile with a symptomatic person subsequently 
Table 1. MERS-CoV symptoms reported by healthcare personnel, King Faisal Specialist Hospital and Research Center, Jeddah, Saudi Arabia, March-July 2014*

\begin{tabular}{lccc}
\hline Symptom & $\begin{array}{c}\text { Seropositive, } \\
\text { no./No.† (\%) }\end{array}$ & $\begin{array}{c}\text { Seronegative, } \\
\text { no./No.† (\%) }\end{array}$ & p value \\
\hline Muscle pain & $13 / 20(65.0)$ & $66 / 260(25.4)$ & 0.0001 \\
Fever & $12 / 19(63.2)$ & $42 / 258(16.3)$ & $<0.0001$ \\
Dry cough & $11 / 20(55.0)$ & $80 / 262(30.5)$ & 0.02 \\
Headache & $11 / 20(55.0)$ & $80 / 262(30.5)$ & 0.02 \\
Diarrhea & $7 / 20(35.0)$ & $21 / 262(8.0)$ & 0.0001 \\
Nausea & $7 / 20(35.0)$ & $18 / 262(6.9)$ & $<0.0001$ \\
Shortness of breath & $7 / 20(35.0)$ & $32 / 261(12.3)$ & 0.005 \\
Runny nose & $6 / 19(31.6)$ & $92 / 263(35.0)$ & 0.76 \\
Chills & $6 / 20(30.0)$ & $23 / 261(8.8)$ & 0.003 \\
Sore throat & $5 / 20(25.0)$ & $118 / 263(44.9)$ & 0.08 \\
Vomiting & $4 / 20(20.0)$ & $10 / 262(3.8)$ & 0.01 \\
Productive cough & $3 / 18(16.7)$ & $39 / 263(14.8)$ & 0.74 \\
Rash & $1 / 20(5.0)$ & $4 / 259(1.5)$ & 0.26 \\
None & $3 / 20(15.0)$ & $94 / 263(35.7)$ & 0.019 \\
\hline *MERS-CoV, Middle East respiratory syndrome coronavirus. & \\
†Denominator is the number of healthcare personnel who responded to \\
the question.
\end{tabular}

confirmed to have MERS-CoV infection. We therefore limited our analysis of risk factors, including PPE use, to any study participant who reported direct contact (i.e., within 2 meters) with MERS-CoV patients in the hospital (Table 2, http://wwwnc.cdc.gov/EID/article/22/11/16-0920-T2. $\mathrm{htm})$. Total time spent in a MERS-CoV patient's room or handling the patient's bedding, equipment, or fluids did not significantly differ between seropositive and seronegative HCP $(p=0.93)$, nor did the number of MERS-CoV patients cared for during the study period (median 3.0 and 5.0 patients for seropositive and seronegative HCP, respectively; $p=0.75$ ). We found no association between animal contact and infection.

We assessed HCP's self-reported use of PPE during care of MERS-CoV patients, stratified by type of equipment and type of patient interaction (Table 3). HCP who reported always covering their nose and mouth with either a medical mask or N95 respirator had lower risk for infection than did HCP reporting not always or never doing so, although this association was statistically significant only among HCP present in the room where aerosol-generating procedures were conducted. HCP who reported always using a medical mask for direct patient contact were $\approx 3$ times more likely to have MERS-CoV infection than were HCP who reported not always or never using a medical mask (98\% of whom reported always or sometimes using an N95 respirator), a trend that was not statistically significant ( $\mathrm{p}=$ 0.10). Conversely, those who reported always using N95 respirators for direct patient contact were less likely to be seropositive, a trend that approached statistical significance $(\mathrm{p}=0.07)$.

Because medical mask and N95 respirator use were strongly and inversely correlated, we built separate multivariate models, one that assessed risk for medical mask use (model 1) and another that assessed risk for N95 respirator use (model 2). In both models, having participated in infection control training that included information about MERS$\mathrm{CoV}$ prevention was associated with a significant and strong protective effect, and there was a strong but statistically insignificant trend toward increased risk among smokers. In model 1, HCP who reported always using a medical mask for direct MERS-CoV patient care were significantly more likely to be seropositive than those who reported not always or never wearing a medical mask (almost all of whom sometimes or always wore an N95 respirator) (relative risk [RR] $2.73,95 \%$ CI $0.99-7.54)$. This model also included past or current smoking (RR 2.54, 95\% CI 0.93-6.96) and participation in MERS-CoV infection control training (RR 0.28, $95 \%$ CI $0.10-0.80)$. In model 2, N95 respirator use was associated with a strong protective trend; HCP who always used an N95 respirator for direct MERS-CoV patient care were $56 \%$ less likely to be seropositive than were those who reported not always or never using an N95 respirator (almost all of whom sometimes or always wore a medical mask) (RR 0.44, 95\% CI 0.15-1.24). This model also included past or current smoking (RR 2.51, 95\% CI 0.92-6.87) and participation in MERS-CoV infection control training (RR $0.33,95 \%$ CI $0.12-0.90)$.

\section{Discussion}

We report this seroepidemiologic study to quantify the risk for MERS-CoV infection among HCP. The findings have important implications for infection control practice. Our results suggest that the attack rate of MERS-CoV infection among healthcare workers is substantially higher than that in previous reports that used nonserologic methods of detection (15-17). The spectrum of illness appears to be broader than previously described; infection caused a relatively mild illness in most cases. Infections occurred almost exclusively among HCP having close contact with a MERS-CoV patient.

Most HCP in this cohort reported always covering their nose and mouth with a medical mask or N95 respirator when caring for a MERS-CoV patient, which appeared to protect against infection among HCP participating in aerosol-generating procedures. When we stratified by type of mask, we observed an increased risk for MERS-CoV infection among HCP who reported always using medical masks and, conversely, a lower risk among those who reported always using N95 respirators. Taken together, these results raise the hypothesis that short-range aerosol transmission might have factored in transmission. Previous studies suggest that some respiratory viruses (e.g., influenza, severe acute respiratory syndrome coronavirus, rhinovirus) that are transmitted primarily by droplets and/or contact might simultaneously be spread through aerosol under certain conditions and perhaps by certain patients $(18-22)$. Aerosol transmission in close proximity to the patient might not 
Table 3. PPE used by healthcare personnel during care of MERS-CoV patients, King Faisal Specialist Hospital and Research Center, Jeddah, Saudi Arabia, March-July 2014*

\begin{tabular}{|c|c|c|c|c|}
\hline PPE used, contact type & $\begin{array}{c}\text { Always wore PPE, } \dagger \\
\text { no. seropositive/total } \ddagger(\%)\end{array}$ & $\begin{array}{c}\text { Sometimes/never wore PPE,§ } \\
\text { no. seropositive/total } \ddagger(\%)\end{array}$ & $\operatorname{RR}(95 \% \mathrm{Cl})$ & $p$ value \\
\hline Gloves & $18 / 197(9.1)$ & $0 / 21(0)$ & NA & NA \\
\hline Gown & $11 / 139(7.9)$ & $7 / 79(8.9)$ & $0.89(0.36-2.21)$ & 0.81 \\
\hline \multicolumn{5}{|l|}{ Eye protection } \\
\hline Direct contact & $1 / 47(2.1)$ & $17 / 165$ (10.3) & $0.21(0.03-1.51)$ & 0.13 \\
\hline Aerosol-generating procedure & $3 / 62(4.8)$ & $11 / 100(11.0)$ & $0.44(0.13-1.51)$ & 0.25 \\
\hline \multicolumn{5}{|c|}{ Covering of nose and mouth with medical mask or N95 respiratorT } \\
\hline Direct patient contact & $11 / 151(7.3)$ & 7/66 (10.6) & $0.69(0.28-1.69)$ & 0.43 \\
\hline Aerosol-generating procedures & $8 / 133(6.0)$ & $6 / 32(18.8)$ & $0.32(0.12-0.86)$ & 0.03 \\
\hline \multicolumn{5}{|l|}{ Medical mask } \\
\hline Direct patient contact & 9/69 (13.0) & 9/142\# (6.3) & $2.06(0.86-4.95)$ & 0.10 \\
\hline Aerosol-generating procedures & $5 / 81(6.2)$ & $8 / 76(10.5)$ & $0.59(0.20-1.71)$ & 0.39 \\
\hline \multicolumn{5}{|l|}{ N95 respirator } \\
\hline Direct patient contact & $6 / 116(5.2)$ & $12 / 101^{* *}(11.9)$ & $0.44(0.17-1.12)$ & 0.07 \\
\hline Aerosol-generating procedures & $5 / 90(5.6)$ & $9 / 73(12.3)$ & $0.45(0.16-1.29)$ & 0.16 \\
\hline \multicolumn{5}{|c|}{$\begin{array}{l}\text { *MERS-CoV, Middle East respiratory syndrome coronavirus; NA, not applicable; PPE, personal protective equipment; RR, relative risk. } \\
\text { tReported always wearing PPE indicated in table row when caring for MERS-CoV patients. } \\
\text { tTotal number of healthcare personnel who responded to the question about PPE. } \\
\text { §Reported not always or never wearing PPE indicated in table row when caring for MERS-CoV patients. } \\
\text { đReported use of medical mask and N95 respirator were not mutually exclusive categories; therefore the number of healthcare personnel reporting } \\
\text { always wearing either an N95 respirator or always wearing a medical mask does not sum to the "covering of nose and mouth with medical mask or N95 } \\
\text { respirator" category. } \\
\text { \#Of the } 142 \text { who reported not always or never wearing a medical mask, } 139(98 \%) \text { reported always or not always wearing an N95 respirator (55\% always, } \\
45 \% \text { not always). } \\
\text { **Of the } 101 \text { who sometimes or never wore an N95 respirator, } 96(95 \%) \text { reported always or not always wearing a medical mask ( } 35 \% \text { always, } 65 \% \text { not } \\
\text { always). }\end{array}$} \\
\hline
\end{tabular}

necessarily be accompanied by long-range transmission because the risk for such transmission might be affected by the infectious dose, the amount of aerosolized particles generated at the source, and the rate of biologic decay of the agent (22). We found no evidence of long-range aerosol transmission. Until additional information about the mode of MERS-CoV transmission is available, it seems prudent to take precautions against aerosol spread in healthcare settings when feasible to do so.

The combined attack rate for HCP who worked in units known to house patients with MERS-CoV infection (8\%) was substantially higher than that in previous studies, which described attack rates for HCP of $\leq 1 \%(15-17)$. These prior studies did not use serologic methods to detect infection but rather relied on rRT-PCR of nasopharyngeal swabs. All 20 seropositive HCP in our study were screened with nasopharyngeal swabs, and only $5(25 \%)$ of these tests showed evidence of MERS-CoV by rRT-PCR. Therefore, screening for viral shedding using nasopharyngeal swabs might be an insensitive method for detecting infection, perhaps because of variability in timing of samples in relationship to exposure, and studies relying solely on this method of case detection might underestimate attack rates.

Our study suggests that almost all MERS-CoV infection among HCP occurs among those having close contact with patients known to be infected with MERS-CoV. We observed the highest attack rates among radiology technicians, followed by nurses. We hypothesize that radiology technicians most likely were exposed while obtaining portable chest radiographs, a procedure that requires close contact (e.g., positioning the patient for cassette placement) with patients who might be likely to have worsening respiratory status and be highly contagious. We identified no seropositive HCP who worked in the unit not known to house any MERS-CoV patients, suggesting that the background rate of MERS-CoV infection among HCP was low in the absence of known exposure to infected patients and that the virus was not circulating widely among staff.

HCP who had undergone infection control training specific to MERS-CoV had a lower risk for infection. This finding underscores the critical need for adequate infection control training, especially in settings with ongoing transmission of epidemiologically important pathogens.

We observed a broad spectrum of illness among HCP, and in most cases illness was relatively mild. Most illnesses were characterized by myalgia, fever, headache, and dry cough. Gastrointestinal symptoms were present in $50 \%$ of infected HCP; and 3 (15\%) reported no symptoms. Most seropositive HCP with symptoms sought care, but only a small minority were recognized as having MERS-CoV infection. All 20 infected HCP survived, and only 5 required hospitalization. The spectrum of illness we observed was broader than that described in previous case series of MERS-CoV infection $(15,23,24)$, which probably were biased toward identifying patients with more severe illness because testing for MERS-CoV infection has largely been triggered by case definitions requiring evidence of pneumonia (10). The observation that most MERS-CoV infections among HCP are likely to be relatively mild and unrecognized has potentially important implications for 
infection control practice. Although little is known about risk for transmission from persons with mild MERS-CoVinfection, HCP with unrecognized MERS-CoV infection might be a reservoir for transmission to hospitalized patients who are more susceptible to severe illness because of underlying illnesses. Transmission from persons with unrecognized MERS-CoV infections might have contributed to the major role healthcare-associated transmission has played in the epidemiology of MERS-CoV $(6,8,9)$. Thus, control of transmission in healthcare settings might depend on maintaining a low threshold for suspicion of MERSCoV infection among exposed HCP and other persons with a relatively mild viral syndrome.

Our study did not identify strong associations with underlying chronic illnesses, most likely because the prevalence of such conditions was low $(<10 \%)$ in this population. HCPs with a history of smoking had a risk for infection almost 3 times that of nonsmokers. We found no association between MERS-CoV infection and sex. Most case series to date have demonstrated a male predominance among casepatients $(15,23,24)$, but our study suggests this association might be explained by social and behavioral factors that increase exposure to MERS-CoV, rather than a sex-specific difference in biological susceptibility.

Our study has several strengths. We compared MERS$\mathrm{CoV}$ infected and uninfected HCP to determine risk factors for acquiring infection during patient care. The use of serologic testing to determine infection status enabled unbiased case ascertainment, an examination of the full spectrum of disease, and a comparison of the risks associated with a wide range of specific patient care activities.

Our study also has limitations. First, questionnaires were administered several weeks after possible exposures, and therefore the potential exists for recall bias. Recall bias can limit assessment of important variables, such as frequency of exposure and duration of contact during specific procedure. However, HCP and interviewers were unaware of their serologic status at the time of interview; their answers would not have been influenced by knowledge of these results. Moreover, symptoms of illness were unlikely to have introduced systematic bias to responses because most uninfected and infected groups reported illness. Second, we used only 1 serum sample for serologic testing. Because of the retrospective nature of our study, baseline serologic tests were not conducted, and therefore the potential exists for false-positive results. However, seroprevalence of MERS-CoV antibodies in Saudi Arabia is low (0.15\%), making misclassification bias unlikely (25). Third, infected asymptomatic HCP could serve as a potential source of infection to other HCP. Given the retrospective nature of our study, we were not able to characterize these potential exposures. Fourth, as is common with early studies of emerging infectious diseases, sufficiently powering studies can be difficult. Whether negative findings were true null findings or due to small sample sizes is unclear.

In conclusion, we report results of a seroepidemiologic study to quantify risk for MERS-CoV infection among HCP. The attack rate appears to be substantially higher than that in prior reports that used nonserologic methods of detection. Infection in this population most often results in mild illness that might be overlooked; programs to identify and exclude ill HCP who have been exposed to patients with MERS-CoV might help eliminate this reservoir for transmission. Our findings also suggest N95 respirators might be more protective against MERS$\mathrm{CoV}$ infection while in close contact with an infected patient and highlight the possible role of short-range aerosol transmission of MERS-CoV in healthcare settings. Education about standard and MERS-CoV infection control practices appears to be protective, suggesting that adherence to basic practices can effectively prevent MERS$\mathrm{CoV}$ infection among HCP.

\section{Acknowledgments}

We thank and acknowledge the help of staff at King Faisal Specialist Hospital and Research Center-Jeddah, Richard Sanvictores, Shareefah Mossairy, Mohammad Alhroub, Hadeel Ghurab, Sawsan Bassi, Haneen Al Omar, Lubna Alsalmi, Halah AL-Enizi, Lourdes Gozum, Surekha Pankajakshan, Rhonwyn Tighe, Medalin Sundaresan, Alanood Abdulfattah, Maricel Garcia, Melanie Dagalea, Jaffar Khiariy, and Nabeela Albaz. We also thank Azaibi Tamin, Jennifer L. Harcourt, Hayat Caidi, Congrong Miao, and Suvang Trivedi for providing laboratory testing support and Heidi Gruhler, Glenn Abedi, John Watson, Sue Gerber, and Matthew Maenner for their assistance in conducting the study and preparing the manuscript.

This study was funded by the Ministry of Health of Saudi Arabia and the US Centers for Disease Control and Prevention.

Dr. Alraddadi is a consultant of infectious diseases at King Faisal Specialist Hospital and Research Center, Jeddah, Saudi Arabia. His research interests include Middle East respiratory syndrome and transplant-related infections.

\section{References}

1. World Health Organization. Middle East respiratory syndrome coronavirus (MERS-CoV) [cited 2016 May 1]. http://www.who.int/ emergencies/mers-cov/en/

2. World Health Organization. Middle East respiratory syndrome coronavirus (MERS-CoV): summary of current situation, literature update and risk assessment - as of 5 February 2015 [cited 2015 Feb 5]. http://www.who.int/csr/disease/coronavirus_infections/mers5-february-2015.pdf?ua=1

3. World Health Organization. MERS-CoV in Republic of Korea at a glance as of 29 July 2015 [cited 2015 Jul 29]. http://www.wpro.who. int/outbreaks_emergencies/wpro_coronavirus/en/

4. Azhar EI, El-Kafrawy SA, Farraj SA, Hassan AM, Al-Saeed MS, Hashem AM, et al. Evidence for camel-to-human transmission of 
MERS coronavirus. N Engl J Med. 2014;370:2499-505. http://dx.doi.org/10.1056/NEJMoa1401505

5. Kayali G, Peiris M. A more detailed picture of the epidemiology of Middle East respiratory syndrome coronavirus. Lancet Infect Dis. 2015;15:495-7. http://dx.doi.org/10.1016/S1473-3099(15)70128-3

6. Majumder MS, Rivers C, Lofgren E, Fisman D. Estimation of MERS-coronavirus reproductive number and case fatality rate for the spring 2014 Saudi Arabia outbreak: insights from publicly available data. PLoS Curr. 2014;6:6.

7. Müller MA, Meyer B, Corman VM, Al-Masri M, Turkestani A, Ritz D, et al. Presence of Middle East respiratory syndrome coronavirus antibodies in Saudi Arabia: a nationwide, cross-sectional, serological study. Lancet Infect Dis. 2015;15:629. http://dx.doi.org/10.1016/S1473-3099(15)70090-3

8. Drosten C, Muth D, Corman VM, Hussain R, Al Masri M, HajOmar W, et al. An observational, laboratory-based study of outbreaks of Middle East respiratory syndrome coronavirus in Jeddah and Riyadh, Kingdom of Saudi Arabia, 2014. Clin Infect Dis. 2015;60:369-77. http://dx.doi.org/10.1093/cid/ciu812

9. Oboho IK, Tomczyk SM, Al-Asmari AM, Banjar AA, Al-Mugti H, Aloraini MS, et al. 2014 MERS-CoV outbreak in Jeddah-a link to health care facilities. N Engl J Med. 2015;372:846-54. http://dx.doi.org/10.1056/NEJMoa1408636

10. Scientific Advisory Council of the Saudi Arabia Ministry of Health. Infection prevention/control and management guidelines for patients with Middle East respiratory syndrome coronavirus (MERS-CoV) infection [cited 2014 Dec 8]. http://www.moh.gov.sa/en/CCC/StaffRegulations/Corona/Documents/GuidelinesforCoronaPatients.pdf

11. World Health Organization. Infection prevention and control during health care for probable or confirmed cases of novel coronavirus (nCoV) infection [cited 2013 May 6]. http://www.who.int/csr/ disease/coronavirus_infections/IPCnCoVguidance_06May13. pdf?ua $=1$

12. World Health Organization. WHO statement on the eighth meeting of the IHR Emergency Committee regarding MERS-CoV [cited 2015 Feb 5]. http://www.who.int/mediacentre/ news/statements/2015/8th-mers-emergency-committee/en/

13. Perl TM, Price CS. Orchestrated scientific collaboration: critical to the control of MERS-CoV. Ann Intern Med. 2015;163:313-4. http://dx.doi.org/10.7326/M15-1395

14. Al-Abdallat MM, Payne DC, Alqasrawi S, Rha B, Tohme RA, Abedi GR, et al.; Jordan MERS-CoV Investigation Team. Hospital-associated outbreak of Middle East respiratory syndrome coronavirus: a serologic, epidemiologic, and clinical description. Clin Infect Dis. 2014;59:1225-33. http://dx.doi.org/10.1093/cid/ ciu359

15. Assiri A, McGeer A, Perl TM, Price CS, Al Rabeeah AA, Cummings DA, et al.; KSA MERS-CoV Investigation Team.
Hospital outbreak of Middle East respiratory syndrome coronavirus. N Engl J Med. 2013;369:407-16. http://dx.doi.org/10.1056/NEJMoa1306742

16. Health Protection Agency UK Novel Coronavirus Investigation team. Evidence of person-to-person transmission within a family cluster of novel coronavirus infections, United Kingdom, February 2013. Euro Surveill. 2013;18:20427.

17. Memish ZA, Al-Tawfiq JA, Makhdoom HQ, Al-Rabeeah AA, Assiri A, Alhakeem RF, et al. Screening for Middle East respiratory syndrome coronavirus infection in hospital patients and their healthcare worker and family contacts: a prospective descriptive study. Clin Microbiol Infect. 2014;20:469-74. http://dx.doi.org/10.1111/1469-0691.12562

18. Bischoff WE, Swett K, Leng I, Peters TR. Exposure to influenza virus aerosols during routine patient care. J Infect Dis. 2013;207:1037-46. http://dx.doi.org/10.1093/infdis/jis773

19. Hall CB. InfluenzaVirus: here, there, especially air? J Infect Dis. 2013;207:1027-9. http://dx.doi.org/10.1093/infdis/jis774

20. Loeb M, McGeer A, Henry B, Ofner M, Rose D, Hlywka T, et al. SARS among critical care nurses, Toronto. Emerg Infect Dis. 2004;10:251-5. http://dx.doi.org/10.3201/ eid1002.030838

21. Roy CJ, Milton DK. Airborne transmission of communicable infection - the elusive pathway. N Engl J Med. 2004;350:1710-2. http://dx.doi.org/10.1056/NEJMp048051

22. Tellier R. Aerosol transmission of influenza A virus: a review of new studies. J R Soc Interface. 2009;6(Suppl 6):S783-90. http://dx.doi.org/10.1098/rsif.2009.0302.focus

23. Assiri A, Al-Tawfiq JA, Al-Rabeeah AA, Al-Rabiah FA, Al-Hajjar S, Al-Barrak A, et al. Epidemiological, demographic, and clinical characteristics of 47 cases of Middle East respiratory syndrome coronavirus disease from Saudi Arabia: a descriptive study. Lancet Infect Dis. 2013;13:752-61. http://dx.doi.org/10.1016/ S1473-3099(13)70204-4

24. Memish ZA, Zumla AI, Al-Hakeem RF, Al-Rabeeah AA, Stephens GM. Family cluster of Middle East respiratory syndrome coronavirus infections. N Engl J Med. 2013;368:2487-94. http://dx.doi.org/10.1056/NEJMoa1303729

25. Müller MA, Meyer B, Corman VM, Al-Masri M, Turkestani A, Ritz D, et al. Presence of Middle East respiratory syndrome coronavirus antibodies in Saudi Arabia: a nationwide, crosssectional, serological study. Lancet Infect Dis. 2015;15:559-64. http://dx.doi.org/10.1016/S1473-3099(15)70090-3

Address for correspondence: Tariq A. Madani, Department of Medicine, Faculty of Medicine, King Abdulaziz University, PO Box 80215, Jeddah 21589, Saudi Arabia; email: tmadani@kau.edu.sa

\section{EID SPOTLIGHT TOPIC}

MERS is an illness caused by a virus called Middle East Respiratory Syndrome Coronavirus (MERS-CoV). MERS affects the respiratory system. Most MERS patients developed severe acute respiratory illness with symptoms of fever, cough, and shortness of breath. Health officials first reported the disease in Saudi Arabia in September 2012. Through retrospective investigations, health officials later identified that the first known cases of MERS occurred in Jordan in April 2012. MERS-CoV has spread from people with the virus to others through close contact, such as caring for or living with an infected person. 